\title{
FATORES ASSOCIADOS AOS HÁBITOS ALIMENTARES E AO SEDENTARISMO EM IDOSOS COM OBESIDADE
}

\author{
Manoela Vieira Gomes da Costa ${ }^{1}$ \\ Álisson César Cardoso de Freitas ${ }^{2}$ \\ Mateus Medeiros Leite ${ }^{3}$ \\ Alessandro de Oliveira Silva ${ }^{4}$ \\ Silvana Schwerz Funghetto ${ }^{5}$ \\ Márcio Rabelo Mota ${ }^{6}$ \\ Luciano Ramos de Lima ${ }^{7}$ \\ Marina Morato Stival ${ }^{8}$
}

\begin{abstract}
1 Graduada em Enfermagem pela Universidade de Brasília (UnB). Mestranda em Ciências e Tecnologias em Saúde pelo Programa de Pós-graduação em Ciências e Tecnologias em Saúde da Faculdade de Ceilândia na Universidade de Brasília (UnB). E-mail: manoelaunb@gmail.com.

2 Graduado em Enfermagem pela Universidade de Brasília (UnB). Residente de enfermagem no Programa de Residência Multiprofissional em Urgência e Trauma na Escola de Superior de Ciências da Saúde (ESCS). E-mail: alissoncesar.unb@gmail.com.

3 Graduado em Educação Física pelo Centro Universitário de Brasília (UniCEUB). Mestrando em Ciências e Tecnologias em Saúde pelo Programa de Pós-Graduação em Ciências e Tecnologias em Saúde da Faculdade de Ceilândia na Universidade de Brasília (UnB). E-mail: mateus.edf@outlook.com. 4 Graduado em Educação Física pela Universidade Federal de Santa Maria (UFSM). Doutor em Educação Física pela Universidade Católica de Brasília. Professor dos cursos de Educação Física e Medicina do Centro Universitário de Brasília (UniCEUB) e do curso de Medicina da Faculdades Integradas da União Educacional do Planalto Central (FACIPLAC). E-mail: silva.alessandro.oliveira@gmail.com. 5 Graduada em Enfermagem pelas Faculdades Franciscanas (FAFRA). Doutora em Ciências da Saúde pela Universidade de Brasília. Docente do Curso de Pós-Graduação em Ciências e Tecnologias da Saúde da Faculdade de Ceilândia na Universidade de Brasília (UnB). E-mail: silvana.funghetto@gmail.com. 6 Graduado em Educação Física pela Universidade Católica de Brasília (UCB). Pós-Doutor em Educação Física pela Universidade de Brasília. Coordenador e professor da Pós-Graduação lato sensu em Fisiologia e Nutrição Clínica no Centro Universitário de Brasília (UniCEUB). E-mail: marciormota@gmail.com. 7 Graduado em Enfermagem pelo Centro Universitário UniEvangélica (UniEvangélica). Doutor em Ciências e Tecnologias da Saúde pela Faculdade de Ceilândia na Universidade de Brasilia (UnB). E-mail: ramosl|@unb.br. 8 Graduada em Enfermagem pelo Centro Universitário UniEvangélica (UniEvangélica). Doutora em Ciências e Tecnologias da Saúde pela Faculdade de Ceilândia na Universidade de Brasília. Docente do Curso de Enfermagem da Faculdade de Ceilândia na Universidade de Brasília (UnB). E-mail: marinamorato@unb.br.
\end{abstract}


Objetivo: Avaliar os índices antropométricos e a composição corporal, bem como identificar os fatores associados aos hábitos alimentares e ao sedentarismo em idosos com obesidade. Método: Este estudo transversal foi realizado com 206 idosos com obesidade que foram avaliados com o inquérito domiciliar e avaliação da atividade física com o International Physical Activity Questionnaries (IPAQ) na Unidade de Saúde com medidas antropométricas e impedância elétrica com o aparelho Biodynamics. Considerou-se o teste qui-quadrado de Pearson para avaliar a diferença entre proporções. Teste t independente foi utilizado para comparar médias. Adotou-se $p<0,05$ como significância. Resultados: Dos 206 idosos avaliados, ( $n=122)$ eram do sexo feminino $(59,2 \%)$ e $(n=84)$ do sexo masculino $(40,8 \%)$. Os dados gerais da amostra estudada referentes às características demográficas indicaram que 35,9\% possuem entre 60 a 65 anos, 55,8\% são casados, 51,4\% têm renda de até dois salários mínimos, 80,6\% não tem controle/restrição alimentar, 82,5\% não são tabagistas, 62,6\% não ingerem bebida alcoólica, 93,2\% são sedentários, 57,3\% não tem atividade de lazer e 41,3\% possuem em média um a quatro anos de estudo. Em relação aos hábitos alimentares, foram associados o sexo com consumo de verduras $(p=0,009)$, escolaridade com consumo de frutas ( $p=0,041)$ e verduras $(p=0,006)$ e o estado civil com dieta $(p=0,046)$ e consumo de frutas $(p=0,043)$. Os fatores associados ao sedentarismo em idosos com obesidade foram sexo ( $p=0,031)$, renda $(p=0,002)$ e tabagismo $(p<0,000)$. Conclusão: Conhecer tal realidade também é importante para o mapeamento dos serviços de saúde e planejamento de intervenções.

palavras - chave

Envelhecimento. Obesidade. Saúde. Hábitos Alimentares. Atividade Física.

Ao longo dos anos, a prevalência da obesidade vem aumentando rapidamente, atingindo homens e mulheres de todas as faixas etárias, tornando-se assim um problema de saúde pública no Brasil e no Mundo. A Organização Mundial da Saúde caracteriza como obesidade o acúmulo anormal ou excessivo 
de gordura no organismo que pode ocasionar um comprometimento à saúde. Neste sentido, estima-se, segundo a OMS (2004), que $60 \%$ da população mundial apresentam algum problema relacionado à obesidade; em idosos, $11 \%$ a $49,6 \%$ apresentaram problemas relacionados à obesidade, e índices de sobrepeso de $32 \%$ a 52,3\% (ARGENTA KÜMPEL et al., 2011; BARBIERI; MELLO, 2012).

A obesidade em idosos é entendida como uma desordem complexa e multifatorial associada, entre outros fatores, ao ganho de peso vinculado a quedas na taxa de metabolismo, em consequência da perda de massa muscular, diminuição da prática de atividade física e aumento do consumo de alimentos calóricos (SILVA et al., 2013). Do ponto de vista biológico, o envelhecimento é definido como um processo universal, acumulativo, sequencial, progressivo e não patológico de deterioração do organismo, decorrente de uma complicada rede de fatores associados a mudanças significativas, como na composição corporal, ocasionando alterações que podem interferir na saúde (MENEZES et al., 2013).

Além dos fatores próprios do envelhecimento, a má alimentação, sedentarismo, tabagismo, consumo de álcool, estilo de vida, doenças e alterações hormonais, fatores genéticos, nível socioeconômico, fatores psicológicos e fatores demográficos são influentes para o desenvolvimento da obesidade (BARBIERI; MELLO, 2012). Diversos estudos apontam que o sedentarismo e a prática de maus hábitos alimentares, com baixo aporte nutritivo e grande teor de carboidratos, gorduras e sódio, contribuem diretamente para a ocorrência de um conjunto de doenças crônicas como diabetes, osteoporose, cânceres e doenças cardiovasculares (FIGUEIREDO, 2010; GADENZ; BENVEGNÚ, 2013; ZAITUNE et al., 2007).

É relevante o fomento de estudos que ressaltem a importância da prática de atividade física neste grupo etário, uma vez que irá influenciar diretamente em benefícios físicos e psicossociais (ZAITUNE et al., 2007), assim como estudos acerca dos hábitos alimentares, questões socioeconômicas, avaliação da composição corporal e estado nutricional por meio de métodos eficientes, tais como a densitometria óssea (CORTEZ; MARTINS, 2012; RIGO et al., 2009). Com isso, será possível que profissionais da saúde possam identificar os fatores que podem influenciar diretamente a saúde dos idosos, especialmente em relação à obesidade e suas complicações, como a síndrome metabólica. Assim, estes profissionais podem direcionar a assistência conforme a realidade e vivência da população (ARGENTA KÜMPEL et al., 2011; MENEZES et al., 2013).

Já é consenso na literatura que a obesidade está ligada ao estilo de vida, pois têm estreita relação com a alimentação e a prática de atividade física. Em idosos, é importante a realização de estudos como este, pois a obesidade associada a hábitos inadequados pode aumentar a prevalência de doenças crônicas, 
além de potencializar suas complicações com efeitos negativos na capacidade funcional deste grupo etário (SGARIBOLDI et al., 2016; VAGETTI et al., 2017).

Diante do exposto, esse estudo teve como objetivo avaliar os índices antropométricos e a composição corporal, bem como identificar os fatores associados aos hábitos alimentares e ao sedentarismo em idosos com obesidade.

\section{Métodos}

Constitui-se como estudo transversal e quantitativo, realizado em uma região administrativa (RA) de Brasília, com idosos atendidos em uma Unidade Básica de Saúde (UBS). A RA é considerada a maior do Distrito Federal, com população estimada de 489.351 habitantes no ano de 2015 e, deste total, $16,90 \%$ eram idosos. Os serviços de saúde públicos que atendem a população consistem em: quinze UBS, um Centro de Atendimento Psicossocial Álcool e Drogas (CAPSad), uma Unidade de Pronto Atendimento (UPA) e um hospital de grande porte. A UBS escolhida para esta pesquisa é composta por seis equipes da Estratégia Saúde da Família (ESF). Esta pesquisa foi aprovada pelo Comitê de Ética em Pesquisa da Secretaria do Estado de Saúde do Distrito Federal (SES/DF) com CAAE: 0367215.5.0000.5553 e parecer no 1.355 .211 , no escopo de um projeto maior denominado "Abordagem das Condições Crônicas Não Transmissíveis na Atenção Primária à Saúde".

A amostra atendia aos seguintes critérios de inclusão: ter idade mínima de 60 anos; ser residente no setor de localização da Unidade de Saúde da Família; ser classificado com obesidade na avaliação da composição corporal e aceitar participar da pesquisa assinando o Termo de Compromisso Livre e Esclarecido (TCLE).

Devido a UBS de escolha para a pesquisa ainda não ter realizado o cadastramento de família da área, optou-se pela amostragem por conglomerados. Após a análise do mapa geográfico, observou-se que a região era composta por 22 quadras. Assim, foram visitadas as casas de uma quadra sim e outra não pelos pesquisadores, bolsistas e voluntários do projeto, totalizando 11 quadras. Foram visitadas as casas de 265 idosos e, destes, 259 responderam ao Inquérito Domiciliar. Foram excluídos seis idosos, pois dois não aceitaram participar da pesquisa e quatro tinham deficiências cognitivas relatadas pela família, incapacitando-os de responder às perguntas.

No primeiro contato, foram explicados os objetivos da pesquisa e, em caso de aceite, foi aplicado o Inquérito Domiciliar. O Instrumento de coleta de dados utilizado foi o Inquérito Domiciliar do Projeto SABE (Saúde, Bem-estar e Envelhecimento) da Faculdade de Saúde Pública da Universidade de São Paulo 
com adaptações (LEBRÃO; DUARTE, 2003). Este teve o objetivo de investigar os dados socioeconômicos, os hábitos alimentares e a prática de atividade física. Para avaliação da atividade física foi considerado a versão curta do International Physical Activity Questionnaries (IPAQ), classificando o idoso como sedentário ou ativo. Para a avaliação dos hábitos alimentares, foi utilizado a frequência de consumo alimentar semanal de leite, feijão, verduras e frutas, além da presença/ ausência de dieta alimentar (controle/restrição alimentar). Ressalta-se que foi considerado para este estudo o consumo adequado para aquele que referiu consumir esses alimentos todos os dias ou de quatro a seis dias por semana.

Posteriormente, foi agendada uma data para avaliação do estado nutricional e da composição corporal. O idoso foi informado do preparo necessário para realizar a avaliação da composição corporal que constava: estar em jejum, não ingerir bebida alcoólica dois dias antes da avaliação e não realizar atividades físicas no dia anterior a avaliação.

Para a avaliação, os 259 idosos foram atendidos individualmente e foram adotados os seguintes métodos de avaliação: Antropometria - para obtenção do peso e estatura foram realizadas duas verificações no período da manhã e calculada uma média aritmética. Impedância Bioelétrica - para avaliação da composição corporal foi utilizado o aparelho portátil de quatro frequências (5khz, 50khz, 100khz e 200khz - Biodynamics). Antes do exame, todos foram orientados a esvaziarem a bexiga e ficarem 15 minutos de repouso. Foram classificados com obesidade os idosos do sexo masculino com mais de $27 \%$ de gordura corporal e do sexo feminino com mais de 33\% e com Índice de Massa Corporal (IMC) maior que (27 Kg/m²) (ABESO, 2009; OMS, 2004).

Os dados foram analisados pelo software Package for the Social Sciences (SPSS®) versão 20.0. A análise descritiva foi realizada por frequências absolutas, relativas, as médias e desvio padrão. Teste de Shapiro-Wilk foi utilizado para verificar a normalidade dos dados. Para comparação entre dois grupos foi utilizado o teste $t$ independente, uma vez que as variáveis apresentaram uma distribuição normal. Considerou-se o teste qui-quadrado de Pearson para avaliar a diferença entre proporções. Adotou-se $\mathrm{p}<0,05$ como significância.

\section{Resultados}

Dos 259 idosos avaliados, 53 foram excluídos por não estarem classificados com obesidade. Assim, participaram do presente estudo 206 com obesidade $(69,57 \pm 7,64$ anos). Na tabela 1 , são demonstrados os valores de caracterização da composição corporal e perfil antropométrico da amostra. 
Tabela 1 - Características descritivas de composição corporal e perfil antropométrico dos idosos com obesidade.

\begin{tabular}{ll}
\hline Variáveis & Média \pm dp \\
\hline Peso $(\mathrm{Kg})$ & $79,58 \pm 8,94$ \\
Estatura $(\mathrm{m})$ & $1,59 \pm 0,07$ \\
IMC $\left(\mathrm{Kg} / \mathrm{m}^{2}\right)$ & $31,36 \pm 3,52$ \\
$\mathrm{GC}(\%)$ & $40,79 \pm 4,61$ \\
$\mathrm{GC}(\mathrm{Kg})$ & $32,78 \pm 5,09$ \\
$\mathrm{H}_{2} \mathrm{O}(\%)$ & $74,27 \pm 3,11$ \\
\hline
\end{tabular}

Fonte: Tabela elaborada pelos autores.

Legenda: IMC = Índice de massa corporal; GC = Gordura corporal;

$\mathrm{H}_{2} \mathrm{O}=$ Percentual de água corporal.

Dos 206 idosos, 59,2\% são do sexo feminino e 40,8\% são do sexo masculino. Os dados gerais indicam que (35,9\% possuem entre 60 a 65 anos, 55,8\% são casados, 51,4\% têm a renda de até dois salários mínimos, 80,6\% não fazem dieta alimentar, $82,5 \%$ não são tabagistas, $62,6 \%$ não são etilistas, 93,2\% são sedentários, 57,3\% não tem atividade de lazer e 41,3\% possuem em média um a quatro anos de estudo (Tabela 2).

Tabela 2 - Análise descritiva das características demográficas dos idosos com obesidade.

\begin{tabular}{ll}
\hline Variáveis & $\mathbf{n}(\%)$ \\
\hline Sexo & $122(59,2)$ \\
Feminino & $84(40,8)$ \\
Masculino & \\
Idade & $74(35,9)$ \\
60 a 65 anos & $54(26,2)$ \\
66 a 70 anos & $37(18,0)$ \\
71 a 75 anos & $41(19,9)$ \\
$>75$ anos & \\
Estado civil & $115(55,8)$ \\
Casado & $91(44,2)$ \\
Solteiro/viúvo/divorciado & \\
Renda (em Salário Mínimo) & $57(27,7)$ \\
$<1$ SM & $106(51,4)$ \\
1 a 2 SM & $43(20,9)$ \\
$>2$ SM &
\end{tabular}




\begin{tabular}{ll}
\hline Variáveis & $\mathbf{n}(\%)$ \\
\hline Faz dieta alimentar & \\
Sim & $40(19,4)$ \\
Não & $166(80,6)$ \\
Consumo de cigarros/dia & \\
Nenhum & $170(82,5)$ \\
1 a 5 & $10(4,9)$ \\
6 a 10 & $11(5,3)$ \\
11 a 15 & $3(1,5)$ \\
16 a 20 & $7(3,4)$ \\
$>20$ & $5(2,4)$ \\
Etilista & \\
Sim & $77(37,4)$ \\
Não & $129(62,6)$ \\
IPAQ & \\
Sedentário & $192(93,2)$ \\
Ativo & $14(6,8)$ \\
Lazer & \\
Sim & $85(41,3)$ \\
Não & $6(21,9)$ \\
Escolaridade & \\
Analfabeto & \\
\hline anos & \\
\hline
\end{tabular}

Fonte: Tabela elaborada pelos autores.

Legenda: $\mathrm{n}$ = Número; \% = Porcentagem; SM = Salário mínimo.

Nas variáveis de composição corporal, as mulheres idosas apresentaram maior percentual de massa gorda $(\mathrm{p}<0,000)$ e menor massa magra $(\mathrm{p}<0,000)$ em relação aos idosos do sexo masculino. Para massa gorda (kg), não houve diferença significativa entre os sexo $(p>0,05)$ (Figura 1). 
Figura 1 - Comparação do percentual de gordura, massa gorda e massa magra dos idosos de acordo com o sexo.

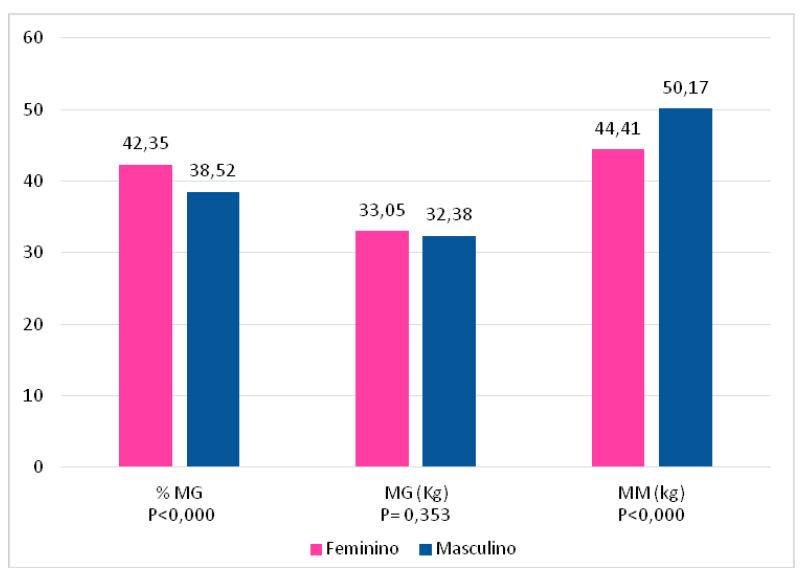

Fonte: Figura elaborada pelos autores.

Legenda: \% MG = Percentual de gordura; MG = Massa gorda; MM = Massa magra; Kg = Quilogramas. Diferença significativa $(p<0,05)$ - Teste t independente.

Na comparação do IMC entre homens e mulheres de acordo com o grupo etário, as mulheres $>75$ anos apresentaram maior IMC em relação aos homens de mesma faixa etária $(\mathrm{p}=0,014)$ (Figura 2).

Figura 2 - Comparação do índice de massa corporal de acordo com o grupo etário e o sexo dos idosos.

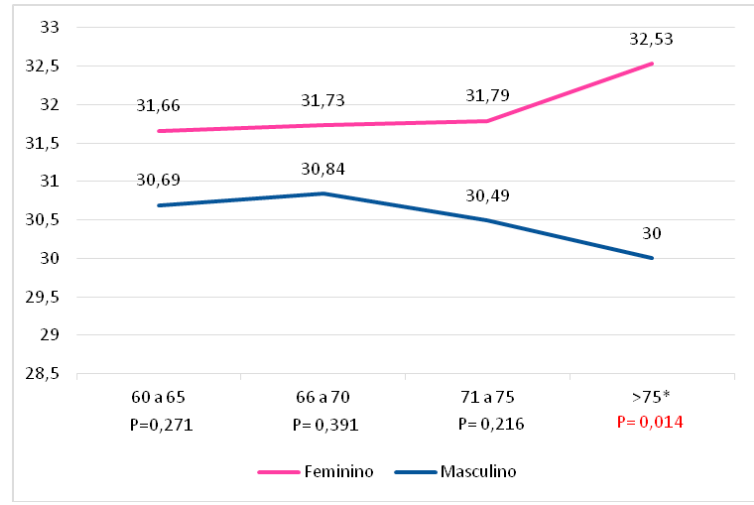

Fonte: Figura elaborada pelos autores.

Legenda: * Diferença significativa $(p<0,05)$ - Teste t independente. 
Em relação aos fatores associados aos hábitos alimentares relacionados à dieta, foi observada uma prevalência de idosos que não fazem dieta/controle alimentar, sendo $84,5 \%$ do sexo masculino e $77,9 \%$ do sexo feminino, idosos com idade entre 60 a 65 anos $(83,8 \%)$, com mais de oito anos de escolaridade $(100 \%)$, casados $(87,0 \%)$, com renda média de um a dois salários mínimos $(83,0 \%)$, aposentados $(83,2 \%)$, sem hábitos tabagistas $(86,7 \%)$ e com hábitos etilistas $(81,4 \%)$ (Tabela 3).

$\mathrm{O}$ sexo foi um fator que influenciou significativamente apenas para o consumo de verduras, sendo o mais adequado relatado pelas idosas do que pelos idosos do sexo masculino $(\mathrm{p}=0,009)$. Já a idade parece não ser um fator associado para influência nos hábitos alimentares dos participantes. Por outro lado, a escolaridade mostrou-se como um importante fator neste contexto. À medida que se aumentava os anos de estudo diminuía-se a prevalência de idosos que afirmavam realizar dieta, porém esta associação não foi significativa $(p>0,05)$. Já em relação ao consumo de frutas e verduras foi observada que à medida que se aumentava os anos de estudos aumentou-se o consumo de frutas $(\mathrm{p}=0,041)$ e verduras $(\mathrm{p}=0,006)$ (Tabela 3$)$.

O estado civil associou-se significativamente ao relato de dieta alimentar e ao consumo de frutas. Idosos que vivem sozinhos como os divorciados e solteiros relataram fazer mais dieta $(\mathrm{p}=0,046)$ e consumirem mais frutas $(\mathrm{p}=$ 0,043 ) do que os casados (Tabela 3).

Fatores como renda, aposentadoria, tabagismo e etilismo não foram significativamente associados à dieta e consumo de frutas e verduras em idosos. Porém observou-se que os idosos com renda maior de dois salários mínimos tiveram maior prevalência de dieta alimentar $(23,3 \%)$ e consomem mais frutas $(79,1 \%)$ e verduras $(62,8 \%)$. Idosos não aposentados fazem mais dieta $(24,0 \%)$ e consomem mais verduras $(61,3 \%)$. Os não tabagistas consomem mais frutas $(67,8 \%)$ e verduras $(62,0 \%)$ do que os tabagistas; aqueles que não são etilistas fazem mais dieta $(19,9 \%)$ e consomem mais verduras $(63,2 \%)$ do que os etilistas (Tabela 3). 
0
0
0
$\square$
$\square$
$\square$

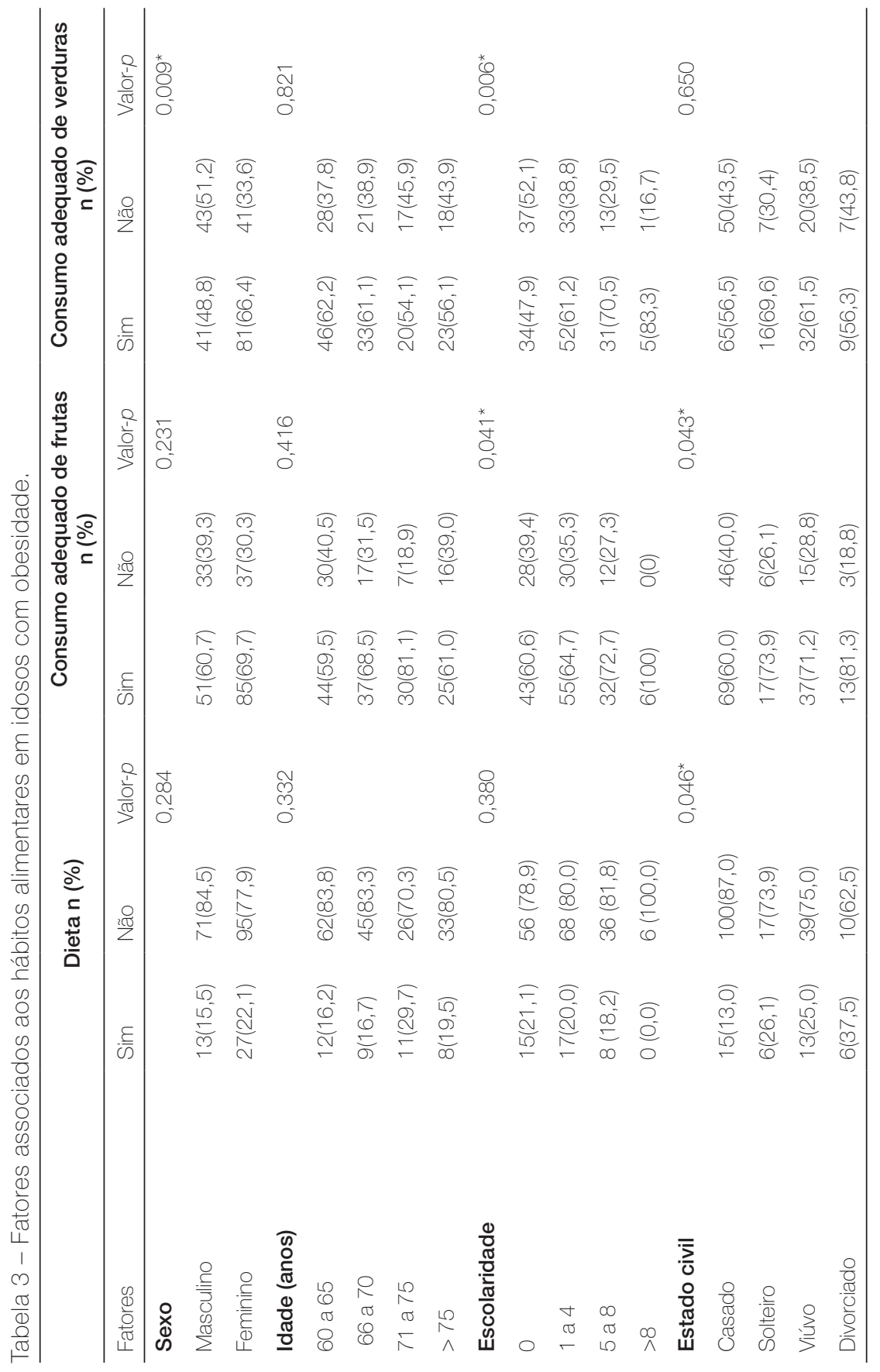




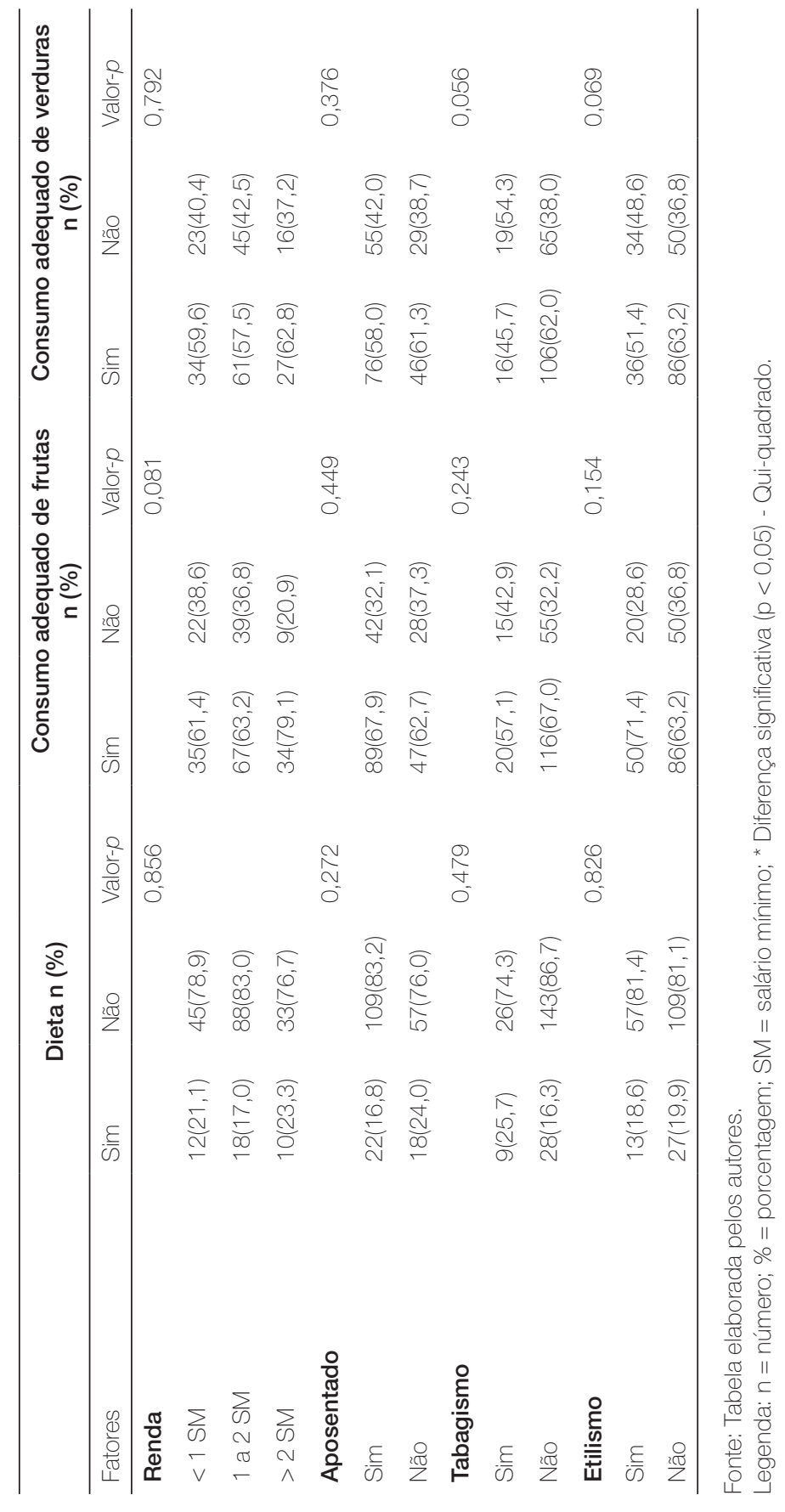

0
0
$\square$
$\square$
$\square$
$\varangle$

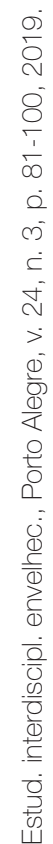


No que diz respeito à prática de atividades físicas observou-se que $93,2 \%$ dos idosos eram sedentários. O sexo, renda e tabagismo foram significativamente associados ao sedentarismo em idosos. Foi evidenciado que idosos do sexo masculino apresentaram maiores índices de sedentarismo $(97,6 \%$; $p=$ 0,031) (Tabela 4).

Em relação à renda média, idosos com um a dois salários mínimos eram mais sedentários do que aqueles com maiores rendas $(97,2 \% ; p=0,002)$. Já em relação ao tabagismo, verificou-se que os idosos tabagistas apresentaram maiores índices de sedentarismo $(94,3 \% ; \mathrm{p}=<0,000)$ (Tabela 4).

A idade, escolaridade, estado civil, aposentadoria e etilismo não foram significativamente associados ao sedentarismo em idosos, porém observou-se maior prevalência de sedentarismo em idosos com idade entre 66 a 70 anos $(98,1 \%)$, analfabetos $(97,2 \%)$, casados e solteiros $(95,7 \%)$, aposentados $(94,7 \%)$, e etilistas (95,6\%) (Tabela 4).

Tabela 4 - Fatores associados à atividade física em idosos com obesidade.

\begin{tabular}{lllll}
\hline & \multicolumn{3}{c}{ Atividade Física } \\
\hline Fatores & $\mathbf{n}(\%)$ & Sedentário & Ativo & Valor- $\boldsymbol{p}$ \\
\hline Sexo & & & & $0,031^{\star}$ \\
Masculino & $84(40,8)$ & $82(97,6)$ & $2(2,4)$ & \\
Feminino & $122(59,2)$ & $110(90,2)$ & $12(9,8)$ & \\
Idade (anos) & & & & 0,229 \\
60 a 65 & $74(35,9)$ & $69(93,2)$ & $5(6,8)$ & \\
66 a 70 & $54(26,2)$ & $53(98,1)$ & $1(1,9)$ & \\
71 a 75 & $37(18,0)$ & $34(91,9)$ & $3(8,1)$ & \\
$>75$ & $41(19,9)$ & $36(87,8)$ & $5(12,2)$ & \\
Escolaridade (anos de estudo) & & & & 0,308 \\
0 & $71(34,5)$ & $69(97,2)$ & $2(2,8)$ & \\
1 a 4 & $85(41,3)$ & $76(89,4)$ & $9(10,6)$ & \\
5 a 8 & $44(21,4)$ & $42(95,5)$ & $2(4,5)$ & \\
$>8$ & $6(2,9)$ & $5(83,3)$ & $1(16,7)$ & \\
Estado civil & & & & 0,108 \\
Casado & $115(55,8)$ & $110(95,7)$ & $5(4,3)$ & \\
Solteiro & $23(11,2)$ & $22(95,7)$ & $1(4,3)$ & \\
Viúvo & $52(25,2)$ & $45(86,5)$ & $7(13,5)$ & \\
Divorciado & $16(7,8)$ & $15(93,8)$ & $1(6,3)$ & \\
\hline
\end{tabular}




\begin{tabular}{lllll}
\hline \multicolumn{5}{c}{ Atividade Física } \\
\hline Fatores & $\mathbf{n}(\%)$ & Sedentário & Ativo & Valor- $\boldsymbol{p}$ \\
\hline Renda & & & & $0,002^{*}$ \\
$<1$ SM & $57(27,7)$ & $55(96,5)$ & $2(3,5)$ & \\
1 a 2 SM & $106(51,5)$ & $103(97,2)$ & $3(2,8)$ & \\
>2SM & $43(20,9)$ & $34(79,1)$ & $9(20,9)$ & \\
Aposentado & & & & 0,775 \\
Sim & $131(63,6)$ & $121(92,4)$ & $10(7,6)$ & \\
Não & $75(36,4)$ & $71(94,7)$ & $4(5,3)$ & \\
Tabagismo & & & & $<0,000^{*}$ \\
Sim & $35(17,0)$ & $33(94,3)$ & $2(5,7)$ & \\
Não & $171(83,0)$ & $159(92,9)$ & $12(7,1)$ & \\
Etilismo & & & & 0,057 \\
Sim & $70(34,0)$ & $62(88,6)$ & $8(11,4)$ & \\
Não & $136(66,0)$ & $130(95,6)$ & $6(4,4)$ & \\
\hline
\end{tabular}

Fonte: Tabela elaborada pelos autores.

Legenda: $n$ = Número; \% = Porcentagem; SM = Salário mínimo; * Diferença significativa $(p<0,05)$ Qui-quadrado.

\section{Discussão}

Com este estudo, é possível perceber que o estado nutricional de idosos compreende alterações decorrentes do envelhecimento que abrangem uma complexa rede de fatores, sendo os principais: fatores socioeconômicos, como sexo, idade, escolaridade, estado civil e renda; e estilo de vida, como tabagismo, alimentação inadequada e sedentarismo. No processo de envelhecimento, essas variáveis podem acarretar alterações físicas que são capazes influenciar na composição corporal do idoso (MENEZES et al., 2013; SOUZA et al., 2016).

É evidenciado também neste estudo, assim como por Menezes et al. (2013) e Cabrera e Jacob-Filho (2001), que o valor médio do IMC entre os idosos é superior nas mulheres, com maior significância acima dos 75 anos, apresentando valores de $32,53\left(\mathrm{~kg} / \mathrm{m}^{2}\right)$ para o IMC feminino e $30\left(\mathrm{~kg} / \mathrm{m}^{2}\right)$ para o IMC masculino. Resultados semelhantes são encontrados em pesquisas nacionais e internacionais, evidenciando maiores prevalências de obesidade em idosos do sexo feminino (ARGENTA KÜMPEL et al., 2011; CABRERA; JACOB-FILHO, 2011; FIGUEIREDO, 2010; NASCIMENTO et al., 2011). 
Já para os homens, o ganho de peso atinge o platô normalmente por volta dos 65 anos e declina em razão dos níveis baixos de testosterona associados ao aumento de gordura abdominal (FIGUEIREDO, 2010). De acordo com Cabrera e Jacob-Filho (2001), nas mulheres, o platô ocorre por volta dos 75 anos, decorrente da diminuição de estrogênio após a menopausa. No Brasil, mulheres apresentam 4,11 vezes mais obesidade em relação ao homens, os quais param de ganhar peso cerca de 10 anos antes, enquanto mulheres continuam tendo um aumento de massa corporal, elevando por conseguinte o seu IMC (MENEZES et al., 2013).

Sabe-se que a obesidade, em idosos, também representa um fator complicador para o seu estado de saúde e ocasiona ou potencializa as complicações advindas das doenças crônicas não transmissíveis (DCNT) associadas ao envelhecimento humano, como predisposição à diabetes mellitus, doenças cerebrovasculares, infarto e aumento do risco de doenças cardiovasculares, diminuição da qualidade de vida, disfunção sexual e sintomas no trato urinário inferior, diminuição da função cognitiva, potencialização das demências e apnéia obstrutiva do sono. A obesidade ainda está associada a diversos tipos de cânceres e causas de morbi-mortalidades (CETIN; NASR, 2014; HAN; TAJAR; LEAN, 2011).

Outra importante complicação da obesidade é a síndrome metabólica, definida como um conjunto de fatores de risco para doença cardiovascular e diabetes, caracterizada por obesidade central, dislipidemia, hiperglicemia e pressão arterial limítrofre, relacionada à obesidade visceral e resistência insulínica. Nos homens, os picos de síndrome metabólica ocorrem entre 50 a 70 anos, enquanto nas mulheres evidenciam-se com 60 a 80 anos, podendo ser prevalente em ambos os gêneros (RIGO et al., 2009; ROCHA; MELO, MENEZES, 2016).

Com relação à idade, há uma associação negativa em relação à diminuição da prevalência de obesidade a partir de 75 anos (SILVEIRA; KAC; BARBOSA, 2009). Em estudo realizado com idosos em Viçosa/MG, por Nascimento et al. (2011) e no Paquistão por Alam et al. (2011), esta diminuição da obesidade com o decorrer da idade é evidenciada apenas nos idosos do sexo masculino, corroborando com os resultados observados no presente estudo, em que mulheres com idade avançada apresentaram maior IMC em relação aos homens de mesma faixa etária.

No presente estudo, em relação aos fatores associados aos hábitos alimentares, o sexo, escolaridade e estado civil foram significativamente associados à dieta e consumo de frutas e verduras em idosos. Em relação ao sexo, observou-se uma melhor alimentação nas mulheres. Tal achado pode ter relação com o fato de que as mulheres estão mais atentas na busca da melhor qualidade de 
vida, mais voltada às práticas de promoção e prevenção da saúde, com maior aderência aos serviços de saúde, dieta saudável e cuidado ao longo da vida (ABREU et al., 2013; CERVATO et al., 2005). Ademais, mulheres idosas apresentam maior consumo adequado de frutas e verduras (hortaliças) (GADENZ; BENVEGNÚ, 2013; VIEBIG, 2010).

A escolaridade foi outro fator que afetou diretamente o consumo de frutas e verduras dos idosos deste estudo. Em outras pesquisas, o consumo adequado de frutas e hortaliças está relacionado intrinsecamente com a escolaridade e renda, uma vez que, quanto maior for o grau de escolaridade e renda, maior será o consumo de frutas e verduras. Com o aumento do grau de escolaridade e renda, houve um aumento nas concepções e conhecimentos acerca de uma alimentação mais saudável, qualidade de vida e acesso a alimentos que tem o aporte nutricional mais saudável, porém que demandam maior subsídio para o acesso (GADENZ; BENVEGNÚ, 2013; VIEBIG, 2010).

Moura e Masquio (2014) apontam à influência da escolaridade na percepção sobre alimentos mais saudáveis, sendo considerado como indispensáveis à alimentação saudável o consumo de frutas e verduras. Também mostra que indivíduos com menor escolaridade tendem a considerar indispensáveis à alimentação, os alimentos de baixo teor nutritivo com grande aporte de carboidratos, gorduras e sódio, na qual o consumo elevado dos mesmos acarreta diretamente no que diz respeito à qualidade de vida e saúde.

Com relação ao estado civil, observou-se que idosos que moram sozinhos faziam mais dieta e apresentavam melhor consumo de frutas e verduras, entretanto não foram encontrados estudos que corroborem tais achados. Acredita-se que tal fato é decorrente da relação de outros fatores socioeconômicos tais como escolaridade e renda e questão de dependência funcional, que vão influenciar diretamente no seu consumo e qualidade de vida e saúde (VIEBIG, 2010).

Ao analisar os fatores associados à atividade física, foi observada uma elevada prevalência de sedentarismo nos idosos participantes. Estudo de Zaitune et al. (2007), também evidenciaram estes resultados, onde o sedentarismo em idosos esteve relacionado a diversos fatores como sexo, faixa etária, grau de escolaridade, estado civil, renda, aposentadoria, tabagismo e etilismo. O sexo, renda e tabagismo foram significativamente associados ao sedentarismo neste estudo. Verificou-se que o comportamento sedentário é prevalente em ambos os gêneros. No entanto, o presente estudo apontou que, no sexo masculino, houve maior prevalência de sedentarismo $(97,6 \%)$.

Por outro lado, Paes et al. (2008) evidenciam maior prevalência de sedentarismo em idosos do sexo feminino, apontando que, culturalmente, as mulheres são menos ativas que os homens. Contudo, o autor relata uma mudança neste 
panorama, na qual as mulheres passaram a adquirir e acumular mais tarefas e atividades, tornando-se mais ativas, enquanto os homens idosos dispõem mais de hábitos considerados não saudáveis (etilismo e tabagismo) do que as mulheres idosas (PAES et al., 2008). Já no estudo de Sousa et al. (2013), as mulheres idosas foram mais ativas em relação aos homens. Assim, o presente estudo mostrou que as idosas são mais ativas, uma vez que as mulheres têm o maior cuidado com a sua própria saúde, destacando a importância da atividade física e alimentação saudável.

No que diz respeito à renda média, ficou evidente a influência da renda no sedentarismo em idosos, pois aqueles que recebem de um a dois salários mínimos (97,2\%) se mostraram mais sedentários do que aqueles com maiores rendas. Tais resultados são evidenciados por outros estudos, como de Zaitune et al. (2007) e Paes et al. (2008). Estes caracterizam a renda como importante fator associado com o sedentarismo, uma vez que influencia diretamente no que diz respeito à atividade física, tempo para atividades e alimentação. Logo, o estudo conclui que idosos com renda mais baixa são mais sedentários, pois, apresentam maiores níveis funcionais, como atividades domiciliares, quando comparados com idosos de renda mais alta.

O aumento do nível funcional acarreta diretamente na disponibilidade de tempo para realizar atividades físicas, tal fato evidenciado pela falta de condição financeira de contratar um profissional para executar atividades em casa e a compra de alimentos mais saudáveis que demandam maior subsídio. Logo, todos esses fatores influenciam diretamente no que diz respeito às práticas de atividades físicas e alimentação (BATISTA; VIEIRA; SILVA, 2012).

Em relação ao tabagismo, foi evidenciada sua influência no sedentarismo em idosos (94,3\%). O tabaco interfere diretamente na expectativa e qualidade de vida, como a prática de atividades físicas e alimentação. A associação do tabaco com o sedentarismo é caracterizada como um fator de risco comportamental que, consequentemente, aumenta o risco de doenças e óbitos em idosos de ambos os sexos tabagistas (FIGUEIREDO, 2010).

Assim, é notável a associação do envelhecimento com Doenças Crônicas Não Transmissíveis (DCNT) e suas consequências, como disfunções na capacidade funcional, cardiovascular, diminuição de força, capacidade cardiorrespiratória associada ao baixo nível de atividade física na população idosa. Torna-se necessário a implementação de programas de exercício, tendo em vista os benefícios de exercícios resistidos com eficiência e segurança para melhora da dor, força muscular, funcionalidade e qualidade de vida, exercícios aeróbios com maior eficiência em promover reduções em escores de depressão de idosos (ANTUNES et al., 2014; NETA et al., 2016). Entretanto, é destacado que 
o estilo de vida ativo e a prática regular de atividades físicas são diferenciais para manutenção da capacidade funcional, composição corporal e parâmetros de força (COELHO et al., 2014; LEITE et al., 2014).

Diante do exposto, observa-se que o idoso apresenta aspectos peculiares que devem ser considerados pelos profissionais que realizam avaliações, como as mudanças na composição corporal relacionadas à idade, que tendem a subestimar a gordura corporal de idosos e que o peso e estatura não conseguem distinguir adiposidade de massa muscular e edema. Neste contexto, baseado nas limitações do emprego isolado do IMC em idosos, é necessário, portanto, o uso de outras medidas de avaliação da composição corporal (RIGO et al., 2009). Além disso, outros fatores devem ser analisados, pois a obesidade vem sendo descrita como uma síndrome mundial, multifatorial e complexa.

Conhecer os fatores que influenciam na prática de atividade física e na alimentação do idoso pode contribuir para adoção de medidas efetivas de promoção da saúde, pois se busca diminuir o excesso de peso neste grupo etário, que é um fator modificável de grande impacto na prevenção e tratamento de doenças crônicas. Além disso, são conhecidos os benefícios que a atividade física e alimentação adequada proporcionam para um envelhecimento saudável.

\section{Conclusão}

Em relação à análise dos índices antropométricos e composição corporal dos idosos, este estudo demonstrou que as mudanças corporais decorrentes do processo de envelhecimento acontecem de forma diferenciada nos homens e mulheres e nas diferentes etapas do envelhecimento. Observou-se que as mulheres com idade mais avançada apresentam maiores valores de massa gordurosa. Os fatores associados aos hábitos alimentares em idosos com obesidade foram sexo, escolaridade e estado civil. Já, em relação ao sedentarismo, foram sexo, renda e tabagismo.

É de suma importância manter-se ampliados os estudos acerca dos fatores relacionados ao sedentarismo e hábitos alimentares na população idosa, uma vez que seus achados poderão influenciar diretamente no direcionamento, intervenção e aplicação de políticas públicas, a fim de melhorar a qualidade de vida, hábitos alimentares e estimular a prática de atividade física de acordo com a realidade socioeconômica e vivência de cada um. Conhecer tal realidade também é importante para o mapeamento dos serviços de saúde de acordo com as necessidades e prioridades da área de abrangência, servindo assim para o norteamento e execução de uma atenção à saúde integral e de qualidade. 


\section{abstract}

Objective: to evaluate anthropometric measurement and body composition, as well as to identify factors associated with eating habits and sedentary lifestyle in the elderly. Method: this cross-sectional study was conducted with 206 obese elderly, assessed with a household survey and physical activity assessment with the International Physical Activity Questionnaires (IPAQ) at the Health Unit with anthropometric measurements and electrical impedance with the Biodynamic appliance. Pearson's chi-square test was considered to evaluate the difference between proportions. Independent t-test was used to compare means. It was adopted $\mathrm{p}<0.05$ as significance. Results: of the 206 elderly evaluated, ( $n=122)$ were female (59.2\%), and $(n=84)$ were male (40.8\%). The general data of the studied sample regarding demographic characteristics indicated that 35.9\% are between 60 and 65 years old, 55.8\% are married, 51.4\% have an income of up to 2 minimum wages, $80.6 \%$ have no control/food restriction, $82.5 \%$ are non-smokers, 62.6\% do not drink alcohol, 93.2\% are sedentary, $57.3 \%$ have no leisure activity and $41.3 \%$ have on average 1 to 4 years of schooling. Regarding eating habits, it was associated the gender with vegetable consumption ( $p=0.009$ ), education with fruit consumption ( $p=0.041)$ and vegetables $(p=0.006)$ and marital status with diet $(p=0.046)$ and dietary intake with fruits $(p=0.043)$. Factors associated with physical inactivity in the obese elderly were gender $(p=0.031)$, income $(p=0.002)$ and smoking $(p<0.000)$. Conclusion: Knowing this reality is also important for health service mapping and intervention planning.

keywords

Aging. Obesity. Health. Eating Habits. Physical Activity.

referências

ABREU, I. C. M. E. et al. Baixa renda entre os idosos participantes da terceira idade está associada à qualidade da dieta. Alimentos e Nutrição Araraquara, São Paulo, v. 24, n. 3, p. 349-357, jul./set. 2013. 
ALAM, I. et al. Relationship between anthropometric variables and nutrient intake in apparently healthy male elderly individuals: a study from Pakistan. Nutrition Journal, v. 10, n. 1, p. 111-117, oct. 2011

ANTUNES, H. et al. O baixo consumo de oxigênio tem reflexos nos escores de depressão em idosos. Revista Brasileira de Geriatria e Gerontologia, Rio de Janeiro, v. 17, n. 3, p. 505-515, jul./set. 2014.

ARGENTA KÜMPEL, D. et al. Obesidade em idosos acompanhados pela estratégia de saúde da família. Texto \& Contexto Enfermagem, Santa Catarina, v. 20, n. 3, p. 271-277, jul./set. 2011

ASSOCIAÇÃO BRASILEIRA PARA O ESTUDO DA OBESIDADE E DA SÍNDROME METABÓLICA (ABESO). Diretrizes brasileiras de obesidade 2009/2010. 3. ed. Itapevi, SP: AC Farmacêutica, 2009.

BARBIERI, A. F.; MELLO, R. A. As causas da obesidade: uma análise sob a perspectiva materialista histórica. Conexões: Revista da Faculdade de Educação Física da UNICAMP, Campinas, v. 10, n. 1, p. 121-141, jan./abr. 2012

BATISTA, N.; VIEIRA, D. J. N.; SILVA, G. M. P. Caracterização de idosos participantes de atividade física em um centro de convivência de Teresina/PI. Enfermagem em Foco, v. 3, n. 1, p. 7-11, 2012

CABRERA, M. A. S.; JACOB FILHO, W. Obesidade em idosos: prevalência, distribuição e associação com hábitos e co-morbidades. Arquivos Brasileiros de Endocrinologia \& Metabologia, São Paulo, v. 45, n. 5, p. 494-501, out. 2001.

CERVATO, A. M. et al. Educação nutricional para adultos e idosos: uma experiência positiva em universidade aberta para a terceira idade. Revista de Nutrição, Campinas, v. 18, n. 1, p. 41-52, jan./fev. 2005.

CETIN, D.; NASR, G. Obesity in the elderly: more complicated than you think. Cleveland Clinic Journal of Medicine, Cleveland, v. 81, n. 1, p. 51-61, jan. 2014.

COELHO, B. et al. Comparação da força e capacidade funcional entre idosos praticantes de musculação, hidroginástica e não praticantes de exercícios físicos. Revista Brasileira de Geriatria e Gerontologia, Rio de Janeiro, v. 17, n. 3, p. 497-504, jul./set. 2014

CORTEZ, A. C. L.; MARTINS, M. C. C. Indicadores antropométricos do estado nutricional em idosos: uma revisão sistemática. Journal of Health Sciences, v. 14, n. 4 p. 271-277, maio 2012.

FIGUEIREDO, A. C. Fatores sociodemográficos, comportamentais e de saúde associados à obesidade em idosos do Distrito Federal. 2010. 135 f. Dissertação (Mestrado em Nutrição Humana) - Faculdade de Ciências da Saúde, Universidade de Brasília, Brasilia, 2010.

GADENZ, S. D.; BENVEGNÚ, L. A. Hábitos alimentares na prevenção de doenças cardiovasculares e fatores associados em idosos hipertensos. Ciência \& Saúde Coletiva, São Paulo, v. 18, n. 12, p. 3523-3533, fev. 2013.

HAN, T. S.; TAJAR, A.; LEAN, M. E. J. Obesity and weight management in the elderly. British Medical Bulletin, v. 97, n. 1, p. 169-196, mar. 2011

LEBRÃO, M.; DUARTE, Y. A. O. SABE: Saúde, Bem-estar e Envelhecimento. O projeto SABE no município de São Paulo: uma abordagem inicial. Brasília, DF: Organização Pan-Americana da Saúde, 2003.

LEITE, L. et al. Comparative study of anthropometric and body composition variables, and functionality between elderly that perform regular or irregular physical activity. Revista Brasileira de Geriatria e Gerontologia, Rio de Janeiro, v. 17, n. 1, p. 27-37, jan./mar. 2014

MENEZES, T. N. et al. Perfil antropométrico dos idosos residentes em Campina Grande/ PB. Revista Brasileira de Geriatria e Gerontologia, Rio de Janeiro, v. 16, n. 1, p. 19-27, jan./mar. 2013. 
MOURA, A. F.; MASQUIO, D. C. L. A influência da escolaridade na percepção sobre alimentos considerados saudáveis. Revista de Educação Popular, Uberlândia, v. 13, n. 1, p. 82-94, jan./jun. 2014

NASCIMENTO, C. et al. Estado nutricional e fatores associados em idosos do Município de Viçosa, Minas Gerais, Brasil. Cadernos de Saúde Pública, Rio de Janeiro, v. 27, n. 12, p. 2409-2418, dez. 2011.

NETA, R. et al. Impacto de um programa de três meses de exercícios resistidos para idosos com osteoartrite de joelhos, da comunidade de Santa Cruz, Rio Grande do Norte, Brasil. Revista Brasileira de Geriatria e Gerontologia, Rio de Janeiro, v. 19, n. 6, p. 950-957, nov./dez. 2016

ORGANIZAÇÃO MUNDIAL DA SAÚDE (OMS). Obesidade: prevenindo e controlando a epidemia global. Relatório da consultoria da OMS. Genebra: Editora Roca, 2004.

PAES, M. J. O. et al. Impacto do sedentarismo na incidência de doenças crônicas e incapacidades e na ocorrência de óbitos entre os idosos do Município de São Paulo. Saúde Coletiva, São Paulo, v. 5, n. 24, p. 183-188, jun. 2008.

RIGO, J. C. et al. Prevalência de síndrome metabólica em idosos de uma comunidade: comparação entre três métodos diagnósticos. Arquivos Brasileiros de Cardiologia, São Paulo, v. 93, n. 2, p. 85-91, fev. 2009.

ROCHA, F. L.; MELO, R. L. P.; MENEZES, T. N. Fatores associados à síndrome metabólica em idosos do interior do Nordeste brasileiro. Revista Brasileira de Geriatria e Gerontologia, Rio de Janeiro, v. 19, n. 6, p. 978-986, nov./dez. 2016.

SGARIBOLDI, D. et al. Influence of body mass index and age on the lung function of obese women. Revista Brasileira de Geriatria e Gerontologia, Rio de Janeiro, v. 19, n. 4, p. 635-641, jul./ago. 2016.

SILVA, A. O. et al. Association of body composition with sarcopenic obesity in elderly women. International Journal of General Medicine, Londres, v. 6, p. 25-29, jan. 2013.

SILVEIRA, E. A.; KAC, G.; BARBOSA, L. S. Prevalência e fatores associados à obesidade em idosos residentes em Pelotas, Rio Grande do Sul, Brasil: classificação da obesidade segundo dois pontos de corte do índice de massa corporal. Cadernos de Saúde Pública, Rio de Janeiro, v. 25, n. 7, p. 1569-1577, jul. 2009.

SOUSA, S. S. et al. Estudo dos fatores sociodemográficos associados à dependência funcional em idosos. Revista de Enfermagem da UFPI, v. 2, n. 1, p. 44-48, jan./mar. 2013.

SOUZA, J. D. et al. Padrão alimentar de idosos: caracterização e associação com aspectos socioeconômicos. Revista Brasileira de Geriatria e Gerontologia, Rio de Janeiro, v. 19, n. 6, p. 970-977, nov./dez. 2016.

VAGETTI, G. C. et al. Associação do índice de massa corporal com a aptidão funcional de idosas participantes de um programa de atividade física. Revista Brasileira de Geriatria e Gerontologia, Rio de Janeiro, v. 20, n. 2, mar./abr. 2017.

VIEBIG, R. F. Consumo de frutas e hortaliças e funcionamento cognitivo em idosos. 2010. 144 f. Tese (Doutorado em Ciências) - Faculdade de Medicina, Universidade de São Paulo, São Paulo, 2010.

ZAITUNE, M. P. et al. Fatores associados ao sedentarismo no lazer em idosos, Campinas, São Paulo, Brasil. Cadernos de Saúde Pública, Rio de Janeiro, v. 23, n. 6, p. 1329-1338, jun. 2007. 